Cite this: Phys. Chem. Chem. Phys., 2011, 13, 14421-14426

\title{
Molecules for organic electronics studied one by one
}

\author{
Jörg Meyer, ${ }^{a}$ Anja Wadewitz, ${ }^{a}$ Lokamani, ${ }^{a}$ Cormac Toher, ${ }^{a}$ Roland Gresser, ${ }^{b}$ \\ Karl Leo, ${ }^{b}$ Moritz Riede, ${ }^{b}$ Francesca Moresco ${ }^{* a}$ and Gianaurelio Cuniberti ${ }^{a c}$
}

Received 31st March 2011, Accepted 18th July 2011

DOI: $10.1039 / \mathrm{c1cp20999j}$

The electronic and geometrical structure of single difluoro-bora-1,3,5,7-tetraphenyl-azadipyrromethene (aza-BODIPY) molecules adsorbed on the $\mathrm{Au}(111)$ surface is investigated by low temperature scanning tunneling microscopy and spectroscopy in conjunction with ab initio density functional theory simulations of the density of states and of the interaction with the substrate. Our DFT calculations indicate that the aza-BODIPY molecule forms a chemical bond with the $\mathrm{Au}(111)$ substrate, with distortion of the molecular geometry and significant charge transfer between the molecule and the substrate. Nevertheless, most likely due to the low corrugation of the $\mathrm{Au}(111)$ surface, diffusion of the molecule is observed for applied bias in excess of $1 \mathrm{~V}$.

\section{Introduction}

Opto-electronically active organic materials offer several advantages over traditional solid-state semiconductors in the fabrication of devices such as solar cells and light emitting diodes including their low cost, low weight, and flexibility. ${ }^{1,2}$ Aza-BODIPY molecules have been known for more than 15 years $^{3}$ and BODIPY derivatives have been recently investigated in more detail for applications as electron donor material for organic solar cells and their optical properties are presently optimized through the synthesis of new compounds. ${ }^{4}$ Here, we use difluoro-bora-1,3,5,7-tetraphenyl-aza-dipyrromethene; the same structure without the BF2 complex was originally synthesized in 1943 as a synthetic dye. ${ }^{5,6}$

There are two key reasons for the interest in aza-BODIPY for applications in organic electronics, especially in solar cells. First of all, these molecules possess a tunable infrared absorption, ${ }^{7-10}$ and can convert a part of the incident light spectrum into electricity that only a few other currently existing organic compounds can. The second reason is their chemical stability, which eases processing requirements during solar cell production. ${ }^{8,9,11}$ There have already been other BODIPY derivatives incorporated in solar cell devices, ${ }^{12-15}$ but these lack the strong infrared absorption behavior of aza-BODIPY.

To better control the properties of the molecule in the final photovoltaic device, it is important to investigate the influence of different environmental factors on the absorption behavior and on the electronic properties of single molecular species or

${ }^{a}$ Institute for Materials Science and Max Bergmann Center of Biomaterials, Dresden University of Technology, 01062 Dresden, Germany.E-mail: francesca.moresco@tu-dresden.de

${ }^{b}$ Institut für Angewandte Photophysik (IAPP), Technische

Universität Dresden, 01062 Dresden, Germany

${ }^{c}$ Division of IT Convergence Engineering, POSTECH, Pohang 790-784, Republic of Korea even single molecules. Several scanning tunneling microscopy (STM) and spectroscopy (STS) investigations of molecules which are of interest for organic photovoltaics have been reported in recent years, especially concerning metal phthalocyanines ${ }^{16-18}$ and fullerene $\mathrm{C}_{60}{ }^{19-23}$ which currently serve as standard materials for photovoltaic heterojunctions, where phthalocyanines act as the electron donor and $\mathrm{C}_{60}$ as the electron acceptor. ${ }^{1}$

Recently, research on the nature of the interaction between single organic dye molecules such as aza-BODiPY and inorganic substrates was reported, showing the relevance of such interactions to the development of dye-sensitized solar cells, which use an organic donor in combination with an inorganic acceptor. ${ }^{24}$ The understanding of the basic mechanisms governing the interactions between organic molecules and inorganic surfaces at the atomic level is essential for further progress in organic electronics, because it allows for the exploitation of the details of the molecular photo-physical properties as well as for the design of new molecules, which can enhance the functionality and stability of these devices.

In this article, we present the results of a combined theoretical and experimental study of single aza-BODIPY molecules deposited on $\mathrm{Au}(111)$. This is the first systematic, single molecule study of the adsorption behaviour of this class of molecules on any substrate. The electronic and geometric structure of the molecule on the surface is investigated using low temperature scanning tunneling microscopy (STM) in conjunction with ab initio density functional theory (DFT) simulations of the density of states (DOS) and of the interaction with the substrate.

\section{Methodology}

Experiments were performed by scanning tunneling microscopy (STM) and spectroscopy (STS) in ultra high vacuum 
(UHV) conditions (base pressure below $1 \times 10^{-10}$ mbar) at a temperature of $5 \mathrm{~K}$. The STM is kept at $5 \mathrm{~K}$ by thermal contact with a liquid $\mathrm{He}$ cryostat and it is completely shielded from radiation by a double screen. The cryogenic pumping preserves the sample surface uncontaminated for several days after preparation.

The $\mathrm{Au}(111)$ surface was cleaned by several cycles of sputtering and annealing at $450{ }^{\circ} \mathrm{C}$ followed by flashing to $550{ }^{\circ} \mathrm{C}$, forming a clean herringbone reconstruction. Aza-BODIPY molecules were evaporated from a Knudsen cell for a few seconds at a temperature of about $220^{\circ} \mathrm{C}$ onto the clean $\mathrm{Au}(111)$ surface kept at room temperature. The sample was then transferred to the STM without breaking the vacuum. Images were taken in constant current mode typically at a bias voltage $V_{\text {bias }}=-0.5 \mathrm{~V}$ and at a tunneling current $\mathrm{I}=0.1 \mathrm{nA}$. Voltages are applied to the sample with respect to the tip.

STS spectra were recorded by measuring the first derivative of the tunneling current $\left(\mathrm{d} I / \mathrm{d} V_{\text {bias }}\right)$. After positioning the tip above a molecule and switching off the feedback loop, the bias voltage was varied over the energy range of interest with a sinusoidal modulation of about $20 \mathrm{meV}$. Using standard lockin detection techniques, the first derivative of the tunneling current was extracted. Before measuring the STS spectrum on the molecule, STS spectra were recorded on the bare $\mathrm{Au}(111)$ terraces. Only those tips showing the Au(111) surface state at $-0.51 \mathrm{~V}^{25}$ were considered purely metallic and used for STS measurements on the molecules.

Calculations were performed using $\mathrm{DFT}^{26,27}$ as implemented in the SIESTA code. ${ }^{28}$ The local-density approximation (LDA) ${ }^{27}$ to the exchange-correlation potential was used, as this has been shown to give more accurate results for the binding geometry of organic molecules on metal surfaces than the generalized gradient approximation (GGA) ${ }^{29}$ This is due to a cancellation of errors in LDA compensating for the failure of these exchange-correlation functionals to properly describe Van der Waal's forces, whereas GGA functionals can significantly underestimate the molecule-substrate interaction. Nevertheless, calculations were also performed for this system using the PBE formulation of GGA, ${ }^{30}$ and the results showed some qualitative differences to LDA which are discussed in detail below. The SIESTA basis set used for the valence orbitals consisted of double-zeta s and double-zeta polarized p orbitals for C, B, N and $\mathrm{F}$; double-zeta polarized s orbitals for $\mathrm{H}$; and double-zeta $\mathrm{s}$ and single-zeta $\mathrm{p}$ and $\mathrm{d}$ orbitals for $\mathrm{Au}$; while the effect of the core states was described by relativistic pseudopotentials. The cut-off radii for the basis orbitals was set using an energy shift of $0.002 \mathrm{Ry}$, which produced values of $2.85 \AA$, $3.33 \AA, 2.50 \AA$, $2.05 \AA, 3.45 \AA$ and $4.13 \AA$ for the $\mathrm{C}, \mathrm{B}, \mathrm{N}, \mathrm{F}, \mathrm{H}$ and $\mathrm{Au} \mathrm{s}$ orbitals, $3.57 \AA$, $4.18 \AA, 3.32 \AA, 2.56 \AA$, and $6.82 \AA$ for the C, B, $\mathrm{N}, \mathrm{F}$ and $\mathrm{Au} \mathrm{p}$ orbitals, and $3.06 \AA$ for the $\mathrm{Au} \mathrm{d}$ orbitals, respectively. The calculations were performed on a grid of $4 \mathrm{k}$-points in each direction in the plane of the surface. The unit cell used for the calculations measured $20.195 \AA$ by $19.992 \AA$ in the plane, and $30 \AA$ perpendicular to the plane. Geometry relaxations were performed using the conjugate gradient routine in SIESTA, with a convergence threshold of a maximum force of $0.05 \mathrm{eV} / \AA$. The gold substrate was modelled by a slab 3 layers thick with periodic boundary conditions. Using up to six layers for the substrate made little qualitative difference to the results. Note that an artificial broadening of $0.1 \mathrm{eV}$ was added to the plots of the orbital-resolved density of states as a function of energy, in order to improve the visibility of the peaks.

\section{Experimental results}

The adsorption of aza-BODIPY on $\mathrm{Au}(111)$ leads to a submonolayer coverage, where single molecules are uniformly distributed on the Au terraces (see Fig. 1(a)). The molecules preferentially adsorb at the fcc domains of the $\mathrm{Au}(111)$ surface reconstruction and no preferred orientation of the molecule is observed. Impurities are normally present at the elbows of the herringbone reconstruction and at step edges. On $\mathrm{Au}(111)$, the preference for the fcc domains is known for many organic molecules. ${ }^{31}$ However, by lateral manipulation ${ }^{32,33}$ we can easily move a single aza-BODIPY molecule from a fcc to a hcp domain and back without changing its conformation.

An enlarged STM image of a molecule is shown in Fig. 1(b). The aza-BODIPY molecule consists of a central BODIPY core (BODIPY = 4,4-difluoro-4-bora-3a,4a-diaza-s-indacene) with four phenyl rings attached and the carbon atom in the mesoposition replaced by nitrogen, as shown in the schematic superimposed on the simulated STM image in Fig. 1(c). In the experimental image (Fig. 1(b)), as well as in the line scans shown in Fig. 2(a,b), the four rings and the central BODIPY core can easily be identified. The fluorine atom on the other hand is
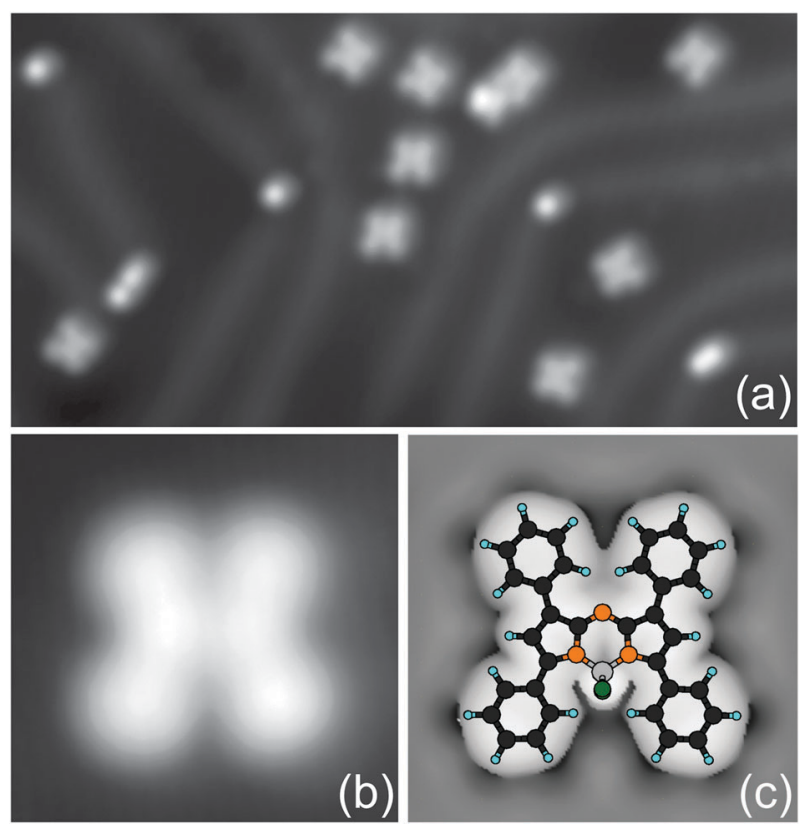

Fig. 1 Aza-Bodipy molecules adsorbed on the $\mathrm{Au}(111)$ surface. (a) Overview STM image showing several adsorbed molecules with different orientation (image size $284 \AA \times 152 \AA$ ). (b) STM image of a single aza-BoDipy molecule (image size $25 \AA \times 25 \AA$ ). (c) Simulated STM image generated from the local DOS (LDOS) at $-0.5 \mathrm{eV}$ calculated with DFT-LDA for aza-BoDIPY on $\mathrm{Au}(111)$ (image size $19.2 \AA \times 19.2 \AA$ ). Schematic of molecule on top of image indicates the calculated position of the molecule. Color code (online version): $\mathrm{C}=$ black (black), $\mathrm{N}=$ light grey (orange), $\mathrm{F}=$ dark grey (green), $\mathrm{B}=$ grey (grey), $\mathrm{H}$ (small) = light grey (blue). 

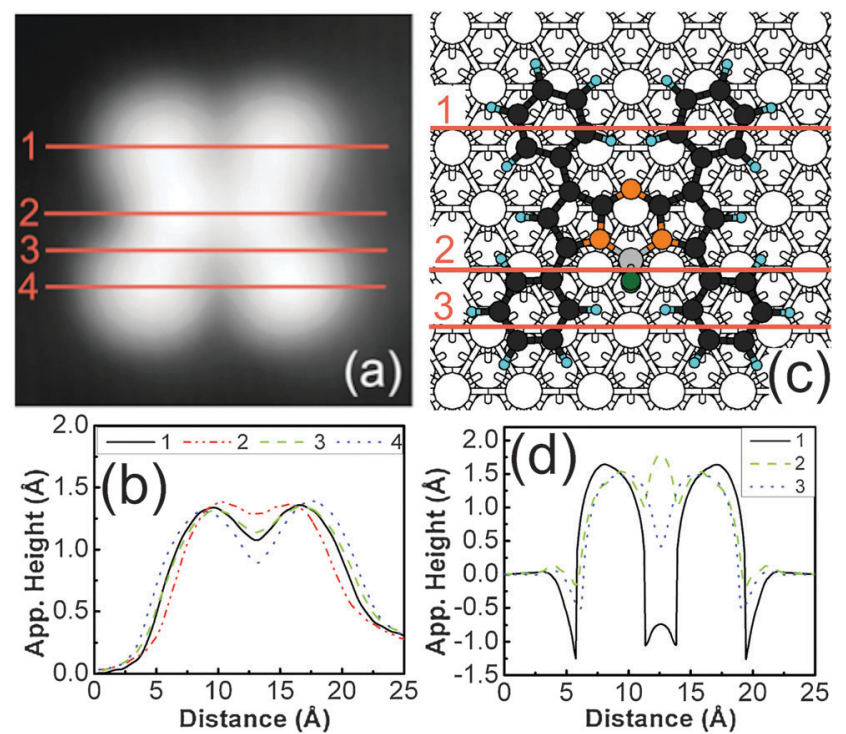

Fig. 2 Line scans over the aza-BodiPy molecule. (a) STM image of aza-BODIPY indicating the positions for the line scans shown in (b) (image size $25 \AA \times 25 \AA$ ). (c) Relaxed geometry for adsorbed aza-BODIPY molecule obtained with DFT-LDA, indicating the positions for line scans shown in (d) (image size $17 \AA \times 17 \AA$ ), which are extracted from the simulated STM image in Fig. 1(c) by plotting sections through the LDOS isosurface.

not visible in the experimental images. This can be clearly seen by comparing the experimental line scans with the theoretical ones in Fig. 2(c,d). No significant differences in the STM images and line scans are observed by varying the tunneling conditions up to a voltage of about $1 \mathrm{~V}$. However, when a bias voltage of $\left|V_{\text {bias }}\right| \geq 1 \mathrm{~V}$ is applied, the molecule starts to diffuse and changes its orientation, as reported in the example of Fig. 3, thus limiting the possible range of STS measurements.

Typical STS spectra measured on the centre of aza-BODIPY molecules and on the bare $\mathrm{Au}(111)$ surface are shown in Fig. 4(a). The spectra recorded on the molecule show a single broad and intense peak centred approximately at $V_{\text {bias }}=$ $0.7 \mathrm{~V}$ corresponding to unoccupied electronic states. Further spectra measured on different positions on the molecule, show similar results and indicate that the energy state observed at $V_{\text {bias }}=0.7 \mathrm{~V}$ is delocalized over the molecule.
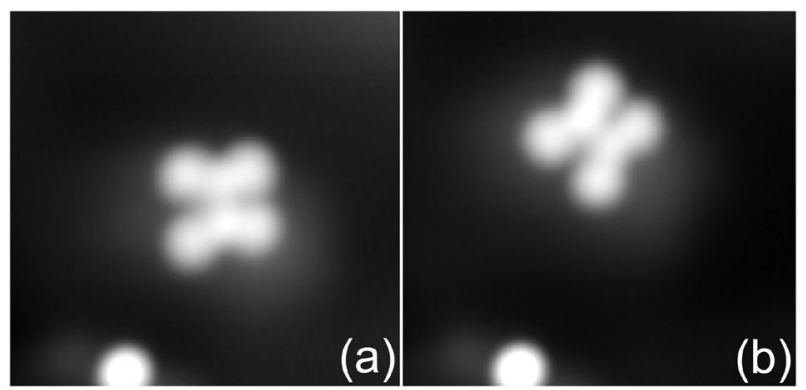

Fig. 3 Diffusion of the aza-BoDipy molecule on the surface at higher bias voltage. STM image (a) before and (b) after application of $V_{\text {bias }}=$ $-1.0 \mathrm{~V}$ (image size $50 \AA \times 48 \AA$ ). As one can see, the molecule moves and rotates on the fcc terrace when imaged at high bias voltage.
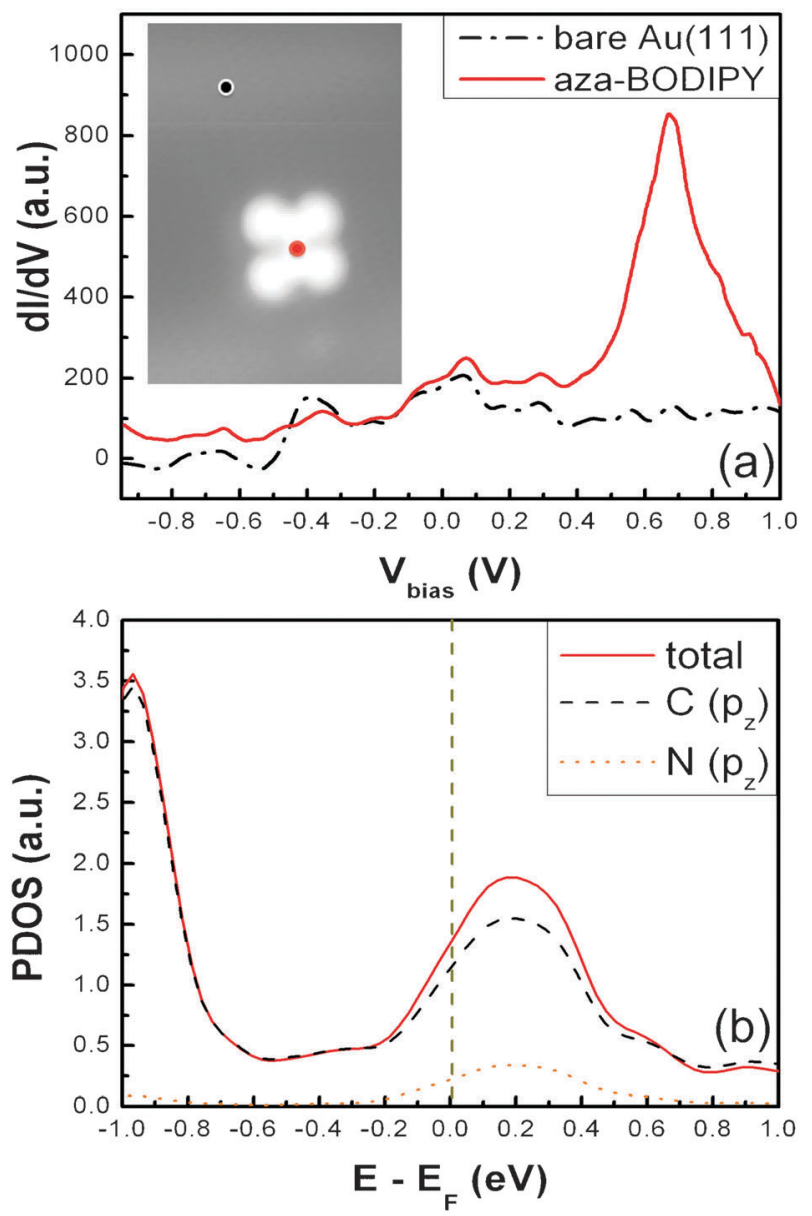

Fig. 4 DOS as a function of energy for adsorbed aza-BODIPY molecule. (a) Experimental DOS, obtained from STS. Inset shows spectroscopy positions (image size $54 \AA \times 88 \AA$ ). (b) Calculated DOS for molecular orbitals obtained with DFT-LDA. Note that the HOMO and LUMO states are primarily constituted of $\mathrm{C}$ and $\mathrm{N} \pi$ orbitals.

\section{Theory results}

The relaxed geometry and orbital-resolved DOS were first calculated with DFT-LDA for the gas-phase aza-BODIPY molecule (see Fig. 5). Both the geometry and the electronic structure were found to be in good agreement with those obtained from calculations using B3LYP exchange-correlation functionals which gives adequate results for various organic molecules. $^{34,35}$ According to both the LDA and GGA calculations described here, the two phenyl rings on either side of the $\mathrm{F}$ atoms are rotated at an angle approximately $21^{\circ}$ to the plane of the BODIPY core, while the rotation angle for the other two phenyl rings is approximately $15^{\circ}$. The Kohn-Sham highest occupied molecular orbital (HOMO) for the isolated gas phase molecule was found in the LDA calculations to have a value of $-5.65 \mathrm{eV}$. For exact Kohn-Sham DFT, $\varepsilon_{\text {HOMO }}=-I P$, where $\varepsilon_{\text {HOMO }}$ is the energy of the Kohn-Sham HOMO and IP is the exact ionization potential. ${ }^{27,36}$ However, in practice, the value of the Kohn-Sham $-\varepsilon_{\mathrm{HOMO}}$ calculated using approximate exchangecorrelation functionals such as LDA is typically several eV less than the true value for the ionization potential. ${ }^{37,38}$ The lowest unoccupied molecular orbital (LUMO) was calculated to have 


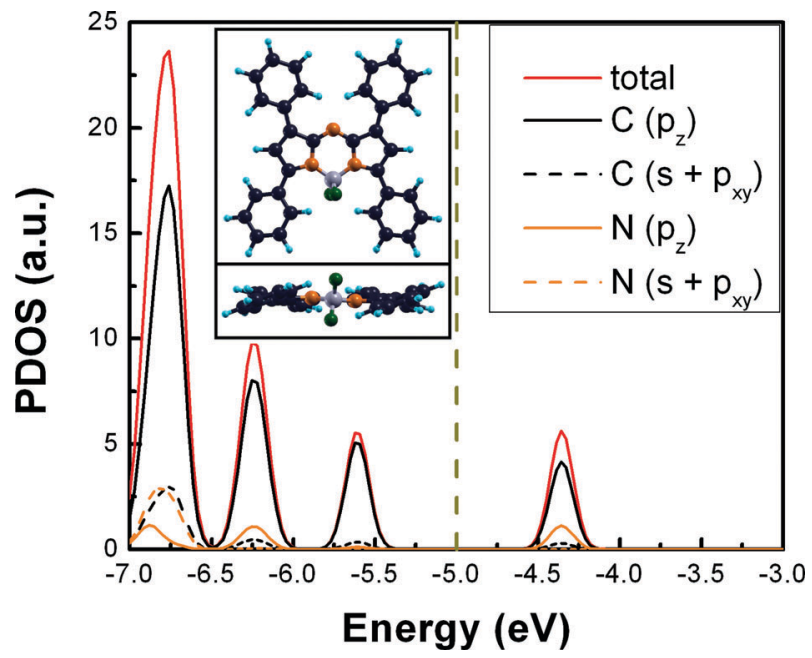

Fig. 5 DOS as a function of energy and structure (inset) for aza-BODIPY molecule in gas-phase. As for the adsorbate phase, the HOMO and LUMO states are primarily constituted of $\mathrm{C}$ and $\mathrm{N}$ $\pi$ orbitals. Note the rotation of the phenyl rings out of the plane of the BODIPY core. The vertical dashed line indicates the division between the occupied and unoccupied states.

an energy of $-4.40 \mathrm{eV}$, although as DFT is a ground state theory the unoccupied Kohn-Sham states do not have a physical meaning in the way that the occupied ones do. The ionization potential was measured experimentally using UPS to have a value of $5.62 \mathrm{eV}$ for a thin film, ${ }^{39}$ although the similarity of this result to the $-\varepsilon_{\text {HOMO }}$ value calculated with LDA is probably coincidental as the gas phase ionization potential would be expected to be somewhat larger than this. The LUMO energy was measured using cyclic voltammetry to have a value of $-4.01 \mathrm{eV}^{39}$ Therefore, DFT-LDA calculations tend to underestimate the size of the HOMO-LUMO gap for this molecule, which is a common problem with LDA calculations, and which needs to be taken into consideration when interpreting the results. Calculations with GGA gave values for the HOMO and LUMO energies of $-5.43 \mathrm{eV}$ and $-4.19 \mathrm{eV}$, respectively, and so give a similar sized HOMO-LUMO gap to LDA. For calculations with both types of exchangecorrelation functionals, the orbital resolved DOS showed that both the HOMO and LUMO orbitals consisted primarily of carbon $\pi$ orbitals which are delocalized over the entire molecule, with a significant contribution also coming from the $\pi$ orbitals on the $\mathrm{N}$ atoms in the BODIPY core.

In order to simulate the situation in the STM measurements, the relaxed geometry for the molecule on a $\mathrm{Au}(111)$ surface was first calculated. The interaction with the surface results in the phenyl rings rotating, so that they are now in the same plane as the BODIPY core. The BODIPY core is slightly raised compared to the phenyl rings, resulting in the molecule assuming a conical shape on surface. The lowest energy configuration was found to be that with the $\mathrm{B}$ atom positioned over a surface bridge site, and the F atom which is closest to the surface positioned over a hollow site. Using LDA, the separation between the $\mathrm{B}$ atom and the surface is found to be $3.33 \AA$, while the average separation between the $\mathrm{C}$ atoms in the phenyl rings and the surface is $2.97 \AA$, with the height of the individual $\mathrm{C}$ atoms above the substrate ranging from $2.83 \AA$ to $3.15 \AA$. The two $\mathrm{F}$ atoms are at heights of $2.17 \AA$ and $4.49 \AA$, with a separation of $2.67 \AA$ between the lower $\mathrm{F}$ atom and the nearest $\mathrm{Au}$ atom. GGA calculations produce a molecule-surface separation which is approximately $0.4 \AA$ to $0.5 \AA$ larger than this.

The effect of the interaction between the surface and the molecule can also be seen in the electronic structure as calculated with LDA, as shown in the orbital resolved DOS in Fig. 4(b). As in the case for the gas phase molecule (see Fig. 5), the frontier orbitals primarily consist of carbon $\pi$ states, but these are now strongly broadened by the interaction with the surface. The GGA calculations showed a much weaker interaction with the substrate, with narrower peaks in the DOS which were shifted to higher energies compared to LDA. By comparing the total energy of the adsorbed molecule/ substrate system to the energies of the separated molecule and substrate, the LDA adsorption energy was determined to be $2.27 \mathrm{eV}$, while that for GGA was only $0.15 \mathrm{eV}$.

\section{Discussion}

Simulated images of the local DOS (LDOS) at $-0.5 \mathrm{~V}$ agree quite well with the measured STM images (see Fig. 1 and 2). In both cases, the LDOS of the molecule appears relatively flat, and with no large peak in the LDOS around the $\mathrm{F}$ atoms at this energy. There is an increase of only about $0.2 \AA$ around the $\mathrm{F}$ atom in the height of the section through the LDOS isosurface as shown in Fig. 2(c), despite the center of this atom lying approximately $1.2 \AA$ above the plane of the molecule. In the line scans from real STM images shown in Fig. 2(b) no protrusion corresponding to the $\mathrm{F}$ atom is visible. In fact, LDA calculations show that the main contribution to the DOS from the states on the $\mathrm{F}$ atoms begins $3.5 \mathrm{eV}$ below the Fermi level, which is far outside the energy range accessible in these experiments. The simulated line scans extracted from the LDOS shown in Fig. 1(c) show that the LDOS isosurface at the molecule is approximately $1.5 \AA$ above that of the $\mathrm{Au}(111)$ surface. This agrees well with the value of approximately $1.4 \AA$ obtained from experimental line scans, and demonstrates that the LDA calculations give an accurate description of the binding geometry of the adsorbed molecule. Both the experimental and calculated line scans are symmetric with broad, flat peaks, again indicating that the phenyl rings rotate during the adsorption process so that they are parallel to the plane of the surface.

Concerning the STS experiment (see Fig. 4(a)) the strong peak observed at around $0.7 \mathrm{~V}$ can be seen in the calculated DOS, although it is shifted towards the Fermi energy and is now centered around $0.2 \mathrm{eV}$ above the Fermi level. This error in the energy value for the LUMO is similar to that for the gas phase molecule which was also seen in other calculations, and again is most likely due to the inability of ground state Kohn-Sham DFT to reliably describe unoccupied states ${ }^{40}$ and the tendency of LDA to underestimate the HOMO LUMO gap. ${ }^{37,38}$ Both the experimental and the calculated LDA LUMO resonances have similar widths, in the range of $0.4 \mathrm{eV}$ to $0.5 \mathrm{eV}$ at half maximum. Calculations with GGA produce a spectrum with narrower resonances due to the 
weakened bonding to the substrate, in which the HOMO and LUMO are both shifted upward in energy by approximately $0.2 \mathrm{eV}$ compared to the LDA results shown in Fig. 4(b), so that they are at approximately $-0.7 \mathrm{eV}$ and $0.5 \mathrm{eV}$, respectively.

During STM measurements, the molecule was observed to diffuse across the substrate when biases of greater than $\pm 1 \mathrm{~V}$ were applied, limiting the measurement of STS spectra to values between $-1 \mathrm{~V}$ and $1 \mathrm{~V}$. In order to understand why the molecule was diffusing on the surface, the total energy differences of different adsorption positions were calculated, with the results shown in Fig. 6. These calculations show that the maximum energy difference between two sites is approximately $0.5 \mathrm{eV}$. The experimental results indicate that the energy transfer between the tunneling electrons and the molecule via inelastic scattering at an applied bias of the order of $1 \mathrm{~V}$ is sufficient to overcome this diffusion potential barrier.

The results of the DFT-LDA calculations suggest that the aza-BODIPY molecule is at least weakly chemisorbed on the $\mathrm{Au}(111)$ surface. The geometry of the molecule is significantly altered during the adsorption process, and the orbitals of the molecule are broadened in energy indicating hybridization with the $\mathrm{Au}$ states. This is somewhat surprising as $\mathrm{Au}(111)$ has a relatively low reactivity compared to other metallic surfaces. Other planar organic dye molecules such PTCDA only physisorb on $\mathrm{Au}(111),{ }^{41}$ although they chemisorb on other metallic surfaces. ${ }^{41,42}$ In order to further investigate the nature of the interaction between aza-BoDIPY and the $\mathrm{Au}(111)$ substrate, the net charge distributions (i.e. the difference between the molecular charge distribution and the superposition of the atomic charge distributions) were plotted for the molecule on the substrate as shown in Fig. 7. This function was also integrated over the Voronoi cell of each atom in a process similar to that of the Voronoi Deformation Density method, ${ }^{43}$ and the results are shown in Table 1 . This shows that in the molecule, the strongly electronegative $\mathrm{F}$ atoms attract charge from the phenyl rings and the B atom, rendering them positively charged. When the molecule is deposited on the surface, charge is transferred from the substrate to the molecule, and the phenyl rings are now slightly negatively charged. It would appear that this charge transfer causes the molecule to weakly chemisorb on the $\mathrm{Au}(111)$

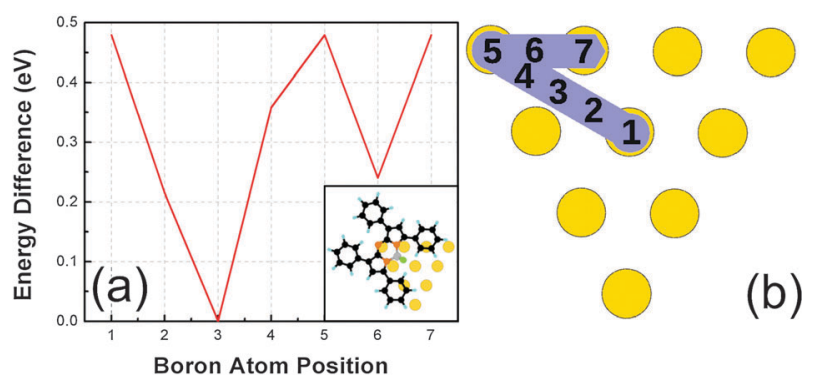

Fig. 6 Total energy differences of an aza-BoDIPY molecule at different adsorption positions. The positions of the $\mathrm{B}$ atom in the molecule relative to the surface lattice are indicated by the numbers in panel (b), which correspond to the energies shown in panel (a). Note that the energy barrier for the molecule to move between sites is $\approx 0.5 \mathrm{eV}$. Inset in (a) shows orientation of the molecule on the surface.

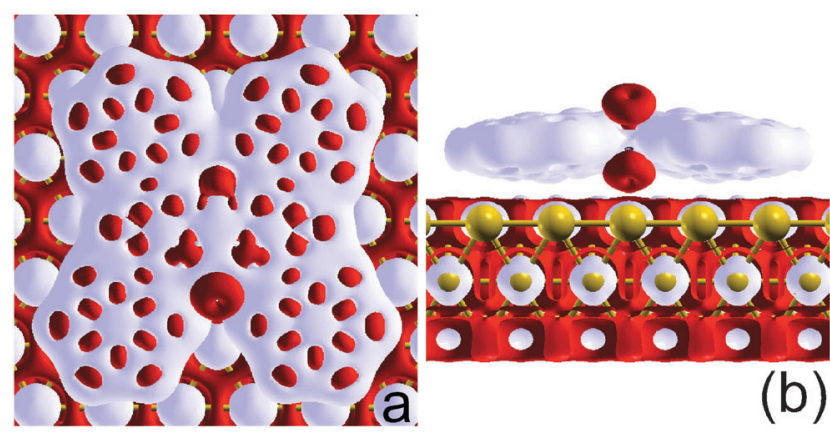

Fig. 7 Charge distribution relative to atomic state for the adsorbed aza-BODIPY molecule shown in (a) top view and (b) side view. Net positive charge is shown in light grey and net negative charge is shown in red (dark grey). Note the polar nature of the molecule, with the electrons concentrating on the electronegative $\mathrm{F}$ atoms.

Table 1 Charge transfer analysis from the DFT-LDA calculations for the valence orbitals of aza-BODIPY molecule both in the gas phase and adsorbed on the $\mathrm{Au}(111)$ substrate. Analysis is performed by integrating the difference between the superposition of the atomic charge densities and the calculated charge density (i.e. the function plotted in Fig. 7) in the Voronoi cell of each atom. Phenyl rings 3 and 5 are the lower phenyl rings, i.e. those on either side of the $\mathrm{F}$ atoms, and rings 1 and 7 are the other, upper phenyl rings, while the "BODIPY core" excludes the $\mathrm{B}$ and $\mathrm{F}$ atoms, which are shown separately. Charge differences are in units of the electronic charge $e$, negative charge indicates extra electrons compared to the atomic state while positive charge indicates fewer electrons

\begin{tabular}{lcc}
\hline Molecule section & Gas phase & Adsorbed \\
\hline Phenyl rings 1, 7 & 0.093 & -0.072 \\
Phenyl rings 3, 5 & 0.133 & -0.038 \\
BODIPY core & 0.006 & -0.139 \\
Boron atom & 0.151 & 0.152 \\
Upper F atom & -0.191 & -0.176 \\
Lower F atom & -0.191 & -0.184 \\
Total & 0.0 & -0.457 \\
\hline
\end{tabular}

substrate. However, it should be noted that it is possible that the LDA calculations are overestimating the extent to which the molecule is charged. While the peak in the calculated DOS corresponding to the molecular LUMO shown in Fig. 4(b) straddles the Fermi level indicating that this orbital is partially occupied, the resonance in the experiments corresponding to this orbital is further from the Fermi level as shown in Fig. 4(a), and so probably contains less charge than would be suggested by the calculations.

\section{Conclusion}

In this paper, we have studied the adsorption and the electronic properties of single aza-BODIPY molecules on $\mathrm{Au}(111)$. Our DFT-LDA results indicate that due to the charge transfer from the Au substrate to the aza-BODIPY molecule, the latter is chemically bound to the surface. The geometry of the adsorbed molecule is weakly distorted compared to the gas phase, assuming a slightly conical shape with the phenyl rings being rotated so that they are aligned almost parallel to the plane of the surface. The STS peak experimentally observed at $V_{\text {bias }} \approx 0.7 \mathrm{eV}$ primarily consists of carbon $\pi$ states broadened by the interaction with the 
surface, with the $\mathrm{F}$ atom not being visible in the STM images due to the contribution to the DOS from the states on this atom being situated at $\approx 3.5 \mathrm{eV}$ below the Fermi level.

To overcome the actual limits of organic electronics in terms of functionality and stability, it is of fundamental importance to understand in detail the properties of the single molecules used in the devices and their interaction with substrates and other molecules. The present work is a first contribution in this direction. Further studies of model systems for organic electronics are planned, where in addition to the moleculesubstrate interaction, the donor-acceptor interaction at the single molecule level will be studied, as well as the influence of monolayer growth on the electronic properties of the molecules. Moreover, the investigation of organic electronics at the atomic level can open new pathways in the development of devices based on a few or even single molecules.

\section{Acknowledgements}

Fruitful discussions with Caroline Gollub, Michael Rohlfing, Viktor Bezugly and Stanislav Avdoshenko are gratefully acknowledged. Funding by the European Union and the Free State of Saxony (ECEMP project A2 (EFRE), ESF project 080942409 InnovaSens), and the ICT-FET Integrated Project AtMol is gratefully acknowledged. RG and MR gratefully acknowledge funding within the BMBF InnoProfile project 03IP602. GC acknowledges support from the South Korean Ministry of Education, Science and Technology (Project WCU ITCE No. R31-2008-00-10100-0). Computational facilities were provided by the Zentrum für Informationsdienste und Hochleistungsrechnen (ZIH) in TU Dresden.

\section{References}

1 M. Riede, T. Mueller, W. Tress, R. Schueppel and K. Leo, Nanotechnology, 2008, 19, 424001.

2 M. C. Gather, A. Köhnen and K. Meerholz, Adv. Mater., 2011, 23, 233.

3 G. Sathyamoorthi, M.-L. Soong, T. W. Ross and J. H. Boyer, Heteroat. Chem., 1993, 4, 603.

4 R. Gresser, M. Hummert, H. Hartmann, K. Leo and M. Riede, Chem.-Eur. J., 2011, 17, 2939-3947.

5 M. A. T. Rogers, Nature, 1943, 151, 504.

6 M. A. T. Rogers, J. Chem. Soc., 1943, 590-596.

7 S. O. McDonnell and D. F. O'Shea, Org. Lett., 2006, 8, 3493-3496.

8 W. Zhao and E. M. Carreira, Angew. Chem., Int. Ed., 2005, 44, $1677-1679$.

9 A. Loudet and K. Burgess, Chem. Rev., 2007, 107, 4891-4932.

10 T. E. Wood and A. Thompson, Chem. Rev., 2007, 107, 1831-1861.

11 R. Ziessel, G. Ulrich and A. Harriman, New J. Chem., 2007, 31, 496-501.

12 S. Erten-Ela, M. D. Yilmaz, B. Icli, Y. Dede, S. Icli and E. U. Akkaya, Org. Lett., 2008, 10, 3299-3302.
13 S. Hattori, K. Ohkubo, Y. Urano, H. Sunahara, T. Nagano, Y. Wada, N. V. Tkachenko, H. Lemmetyinen and S. Fukuzumi, J. Phys. Chem. B, 2005, 109, 15368-15375.

14 D. Kumaresan, R. Thummel, T. Bura, G. Ulrich and R. Ziessel, Chem.-Eur. J., 2009, 15, 6335-6339.

15 T. Rousseau, A. Cravino, T. Bura, G. Ulrich, R. Ziessel and J. Roncali, Chem. Commun., 2009, 1673-1675.

16 G. de la Torre, C. G. Claessens and T. Torres, Chem. Commun., 2007, 2000-2015.

17 M. Moors, A. Krupski, S. Degen, M. Kralj, C. Becker and K. Wandelt, Appl. Surf. Sci., 2008, 254, 4251-4257.

18 X. H. Qiu, G. V. Nazin and W. Ho, Phys. Rev. Lett., 2004, 92, 206102.

19 R. J. Wilson, G. Meijer, D. S. Bethune, R. D. Johnson, D. D. Chambliss, M. S. de Vries, H. E. Hunziker and H. R. Wendt, Nature, 1990, 348, 621-622.

20 C. Joachim, J. K. Gimzewski, R. R. Schlittler and C. Chavy, Phys. Rev. Lett., 1995, 74, 2102-2105.

21 C. Rogero, J. I. Pascual, J. Gomez-Herrero and A. M. Baro, J. Chem. Phys., 2002, 116, 832-836.

22 X. Lu, M. Grobis, K. H. Khoo, S. G. Louie and M. F. Crommie, Phys. Rev. Lett., 2003, 90, 096802.

23 G. Schull, T. Frederiksen, M. Brandbyge and R. Berndt, Phys. Rev. Lett., 2009, 103, 206803.

24 F. De Angelis, S. Fantacci and R. Gebauer, J. Phys. Chem. Lett., 2011, 2, 813-817.

25 W. Chen, V. Madhavan, T. Jamneala and M. F. Crommie, Phys. Rev. Lett., 1998, 80, 1469-1472.

26 H. Hohenberg and W. Kohn, Phys. Rev., 1964, 136, B864.

27 W. Kohn and L. J. Sham, Phys. Rev., 1965, 140, A1133.

28 J. M. Soler, E. Artacho, J. D. Gale, A. García, J. Junquera, P. Ordejón and D. Sanchez-Portal, J. Phys.: Condens. Matter, 2002, 14, 2745.

29 M. Rohlfing and T. Bredow, Phys. Rev. Lett., 2008, 101, 266106.

30 J. P. Perdew, K. Burke and M. Ernzerhof, Phys. Rev. Lett., 1996, 77, 3865.

31 M. Böhringer, K. Morgenstern, W.-D. Schneider, R. Berndt, F. Mauri, A. De Vita and R. Car, Phys. Rev. Lett., 1999, 83, 324-327.

32 L. Bartels, G. Meyer and K.-H. Rieder, Phys. Rev. Lett., 1997, 79, 697-700.

33 F. Moresco, Phys. Rep., 2004, 399, 175-225.

34 J. Tirado-Rives and W. L. Jorgensen, J. Chem. Theory Comput., 2008, 4, 297-306.

35 G. Zhang and C. B. Musgrave, J. Phys. Chem. A, 2007, 111 $1554-1561$.

36 J. P. Perdew, R. G. Parr, M. Levy and J. L. Balduz Jr., Phys. Rev. Lett., 1982, 49, 1691.

37 J. P. Perdew and A. Zunger, Phys. Rev. B: Condens. Matter, 1981, 23, 5048 .

38 C. D. Pemmaraju, T. Archer, D. Sanchez-Portal and S. Sanvito, Phys. Rev. B: Condens. Matter Mater. Phys., 2007, 75, 045101.

39 T. Mueller, R. Gresser, K. Leo and M. Riede, in preparation.

40 R. Baer, E. Livshits and U. Salzner, Аnпи. Rev. Phys. Chem., 2010, 61, 85 .

41 F. S. Tautz, Prog. Surf. Sci., 2007, 82, 479.

42 F. Pump, R. Temirov, O. Neucheva, S. Soubatch, S. Tautz, M. Rohlfing and G. Cuniberti, Appl. Phys. A: Mater. Sci. Process., 2008, 93, 335-343.

43 C. F. Guerra, J.-W. Handgraaf, E. J. Baerends and F. M. Bickelhaupt, J. Comput. Chem., 2004, 25, 189-210. 